\title{
Extraction of Brain Vessels from Magnetic Resonance Angiographic Images: Concise Literature Review, Challenges, and Proposals
}

\author{
Suhuai Luo ${ }^{1}$ and Youliang Zhong ${ }^{2}$ \\ ${ }^{1}$ The School of Design, Communication \& I.T., The University of Newcastle \\ ${ }^{2}$ The School of Computer Science \& Engineering, The University of NSW \\ suhuai.luo@newcastle.edu.au,yzho658@cse.unsw.edu.au
}

\begin{abstract}
The automated extraction of brain vessels from magnetic resonance angiography (MRA) has found its applications in vascular disease diagnosis, endovascular operation and neurosurgical planning. In this paper we first present a concise technical review on cerebral vasculature extraction from MRA. It reveals the latest development in the area of vessel extraction. Then we detail the main challenges to the researchers working in the vessel extraction and segmentation area. Based on the review and our experience in the area, we finally present our proposals on ways of developing robust vessel extracting algorithm. Examples of brain vasculature extracted with advanced hybrid approach are shown. Twenty one references are given.
\end{abstract}

\section{INTRODUCTION}

$\mathrm{M}$ RA [1] is a study on blood vessels with magnetic resonance imaging (MRI). It is an attractive and useful technique for both clinical diagnosis and surgical planning. It is very efficient in detecting narrowing, widening or other abnormalities of arteries or veins in head and neck. Since MRA data provide vessel positions on cross-sectional images only, it is extremely difficult for the clinician to get direct 3D information about vascular connections which are crucial for diagnosing vascular diseases such as stroke. Therefore automatic vessel segmentation and extraction are needed to reveal 3D vessel structures from MRA data. A great amount of effort has been put on the research. Especially, researches in the area are paying great attention to the complexity of MRA images such as large amount of vessels, variable vascular topology and morphology, low intensity contrast between vessels and background, small vessel region comparing to the whole image, etc.

In this paper, we investigate brain vessel extracting techniques, discuss the main challenges to the researchers working in the area, and propose ways of developing robust vessel extracting algorithm. In Section 2, we will brief the characteristics of various basic vasculature segmentation techniques. Then, for each technique, we will review latest progresses. In Section 3, we detail the main challenges to the researchers working in the vessel extraction and segmentation area. Finally, based on the review and our experience in the area, we present our proposals on ways of developing robust vessel extracting algorithm in Section 4.

\section{VESSEL EXTRACTION TECHNIQUES}

There are many ways to classify segmentation techniques for both general applications and specific vasculature extraction [2, 3, 4, 5]. For example, Kirbas et al. [6] divided vessel segmentation techniques into six main categories including pattern recognition, model-based, tracking-based, artificial intelligence-based, neural network-based, and miscellaneous tube-like object detection. Complying with the methodology adopted in [2] and [3], and considering the generality and the latest development in segmentation, we divide vasculature segmentation techniques into four broad categories, namely, edge-oriented, region-oriented, active contour model-based, and hybrid approach. Fig. 1 illustrates the four broad categories, where the numbers indicate the sections describing the content. In the following subsections, each of these techniques will be summarized and illustrated with typical examples.

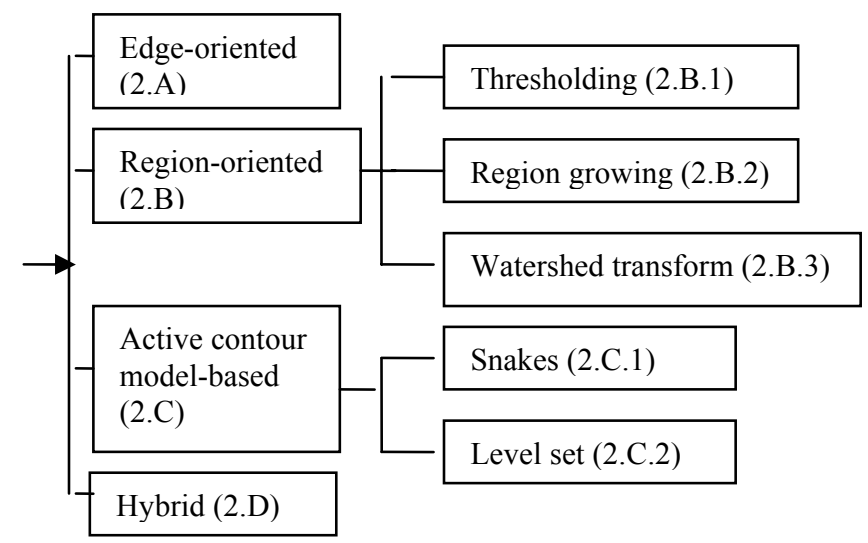

Fig.1 Four categories of vasculature segmentation techniques. Their details can be found in the sections bracketed.

\section{A. Edge-oriented segmentation techniques}

In the approach, edges between regions with different characteristics are first derived with derivative operators such as Sobel and Canny [2, 5, 7]; then regions enclosed by the edges are segmented. The advantages of this technique include: computationally fast, needing non or little priori knowledge about image content. However, its common problem is that edges found are incomplete, resulting unconnected sections of object boundaries. Currently there is no algorithm that can segment vasculature based on edge operators only. Final solution to the problem will reside in applying content-related knowledge and combing various techniques.

\section{B. Region-oriented segmentation techniques}

In this approach, images are segmented into regions of pixels or voxels with certain similarity. There are three 
principal approaches in the category including thresholding, region growing, and watershed.

\section{1) Thresholding-based vessel segmentation}

Thresholding transforms the grey scale values of an image into binary values. Its success depends critically on the selection of an appropriate threshold value. The selection process can be manual, semi-automatic, and automatic. The mostly discussed is the automatic approach which includes (1) discriminant analysis-based; and (2) statistical parameter-based. In (1), the threshold is set based on estimating of the variance of the intensity classes. This will cause difficulties in cerebral vasculature segmentation since vessels occupy very small part of the images therefore its parameters are difficult to get. Method (2) is mostly based on assumptions that each intensity class has a distribution, and the global distribution of the data can be expressed as a linear combination of the distribution of the intensity classes. A typical automatic approach of method (2) is briefed below.

a) Expectation-maximization(EM)-based thresholding

Though EM algorithm itself is not new [8], EM-based automatic thresholding's application in vessel segmentation was developed by Wilson et al. in 1999 [9]. They proposed an adaptive segmentation algorithm for time-of-flight (TOF) MRA data. It first defines a model for the expected distributions found in cerebral TOF MRA data, and then uses a statistical segmentation algorithm that uses the model to automatically extract vessels from the data. The advantages of the algorithm include: simplicity and straightforwardness in use; working well for the arteries surrounding the circle of Willis and for small aneurysms . Its disadvantages are: computationally expensive; for large and giant aneurysms the segmentations may appear to underestimate the aneurysm sac; it is not applicable on small vessels.

\section{2) Region growing-based vessel segmentation}

Region growing starts with a set of pixels called seeds. In the growing process, all the pixels neighboring a seed are examined to see if they are sufficiently similar to the seed according to a homogeneity criterion. If the pixels are similar to a seed, they are added to the region containing the seed. The procedure continues until all the pixels are checked. A limitation of region growing is that it is unstable. Its outcome is sensitive to such facts as the definition of the neighborhood and the choice of homogeneity criterion.

a) locally adaptive region growing algorithm

In 2003, Yi et al. [10] proposed a locally adaptive region growing algorithm for vascular segmentation in $3 \mathrm{D}$ CTA/MRA. In the algorithm, a small local cube is segmented to detect a vessel segment, and the following local cube(s) is determined based on the segmentation result. This procedure is repeated until the segmentation is completed. By confining the segmentation inside each local cube, a robust result can be obtained even in a tubular structure of steadily changing intensity. For segmentation, a locally adaptive and competitive region growing scheme is adopted to obtain well-defined vessel boundaries. It claimed that the method can detect all branches with practically acceptable computational complexity. A good point is that the segmentation result is represented as a tree structure having many branches so that a user may easily correct the result branch-by-branch. According to the observation of the segmented vascular tree examples, a drawback of the method is that only main branches can be segmented.

\section{3) Watershed-based vessel segmentation}

In describing watershed, it is assumed that an image is a landscape where bright pixels represent mountains and dark pixels valleys. Watersheds are the boundaries of objects. Watershed transform denotes a labeling of an image. In the process, all points of a given valley are assigned with a same unique label, and all points of the watersheds are assigned with a special label which is distinct from all the labels of the valleys. Initialization is needed for watershed transform to select at least one seed point for each object. A known drawback of the watershed algorithm is its oversegmentation due to noise or local irregularities in the gradient image.

a) segmentation using watershed and neural networks

Kobashi et al. [11] presented a volume-quantization-based neural network (NN) approach to 3D MRA image segmentation. First a user selected threshold is used to binarise the input and do necessary noise reduction; then watershed segmentation is applied; finally the output of the last step is put into a NN to classify the regions into vessel and non vessel. It studied the building of cerebral vascular tree with examples of segmented tree structure. An advantage of the approach is that other than the selection of initial threshold no much user intervention is involved. Two aspects of shortcoming exist. One is that not many thin branches were shown in the segmented vascular tree. One possible cause could be that the noise reduction stage has deleted small sections of the vessel. Another is that the NN classification rate was about eighty per cent. This will introduce wrong topology structure in practical application.

\section{Active contour model-based vessel segmentation}

In this approach, energy minimizing-based connectivitypreserving relaxation process is applied on images to derive the boundary of an object. Generally speaking, there are two types of active contour models: (a) parametric or explicit models, such as the snakes; (b) geometric or implicit models, such as the level set.

\section{1) Geodesic active contours}

Lorigo et al. [12] proposed a segmentation algorithm based on curve evolution in 3D, also know as "co-dimension two" in geodesic active contours. The method constructs two components for level set equation. The first is mean curvature flow, which is used to derive the Eulerian representation of the level set equation. The second component is the directionality of vessels. The advantages of 
the algorithm are: efficient in segmenting twisted, convoluted, and occluded vessels; and intrinsic computation of vessel radii. Its disadvantages are: needs of manually setting of scale factor; segmented vessels tending to appear thinner then observed; and no validation given.

\section{2) Segmentation using level sets}

A MRA segmentation algorithm with the purpose of extracting the vascular tree was described by Farag et al. [13]. Level sets are initialized with a set of balloons inside the vessels with the largest cross-sectional areas. Then the level sets evolve with time to yield the blood vessels. Good examples of blood vessel extraction on slices and tree structure were given along with validation discussion. It claimed the fat which is around blood vessel and has the same range of intensity as that of blood vessels can be excluded from the segmentation results. However, no details about this were given. It did not say how many initial points were used. The number will decide the degree of automation. It would be good if the paper can provide details of the resultant vascular tree such as the number of tree branches. A similar discussion can be found in paper [14].

\section{Hybrid vessel segmentation}

In this approach, the strengths of several segmentation techniques are combined to achieve segmentation. Basically it integrates the three categories discussed before by using their strength and avoiding their weakness. The trend is that more comprehensive and powerful hybrid segmentation methods are developed.

\section{1) segmentation using $3 D$ multiscale line filter}

Sato et al. [15] proposed to use 3D multiscale line filter (i.e., second derivative calculation) to segment curvilinear structure. The advantages of the method include: it can handle various curvilinear structure; calculation speed is optimized. But main problem with the method is that in selecting volume of interested, user has to select the threshold manually, therefore being susceptible to errors. In practical application, the method can also cause alteration of vessels' radii, vessel disconnection and extra bright dots.

2) segmenting vessel-like patterns using mathematical morphology and curvature evaluation

Zana et al. [16] combined morphology and curvature evaluation to segment. They defined a vessel as a bright pattern of piece-wise connected and locally linear. They singled vessels out of other analogous background patterns by performing a cross-curvature evaluation. The vessels have specific Gaussian-like profiles whose curvature varies smoothly along the crest line. This method is good at using both shape properties and curvature property; and vessels are detected as the only features whose curvature is linearly coherent. Comparing to traditional/standard morphological operators, the geodesic operators used here have the advantages of keeping large vessels completely intact as well as removing small non-vessel objects. Potential problems include: small vessels appear larger because of
Gaussian filtering; disconnection occurred; and some bifurcation points are lost.

3) segmentation using intensity correction, diffusion filtering and region growing

Flasque et al. [17] proposed a method for the detection, representation and visualisation of the cerebral vascular tree on MRA images. The vascular tree is built by iteratively tracking of the vessel centerlines on candidate voxels. The selecting of the candidate voxels is achieved by the combination of intensity correction, diffusion filtering, and region growing. The method can only provide topology information, not morphology. Results of vessel tracking on main vessels only are shown. Fixed thresholding selection in the process may restrict its application.

\section{4) segmentation using intensity ridge \& medialness}

Bullitt et al. [18] segmented vessels by defining seed points, automatically extracting image intensity ridges representing vessel's central skeleton, and determining vessel width with Morse medialness at each skeleton point. Its tree-definition process can automatically exclude erroneous vessel segmentations. The parent-child connectivity information provided by the vessel trees is important to both surgical planning and to guidance of endovascular procedures. A major disadvantage of the method is that as too many seed points may need be manually selected at the beginning.

5) segmentation using differential histogram and seeded region growing

Tuduki et al. [19] presented an automated seeded region growing algorithm to extract blood vessels from MRA. It first thresholds data, then gets the skeleton using thinning, finally does region growing with the skeletons as seeds. A novel aspect of the method is that an optimal threshold value was calculated automatically from the differential histogram using a discriminate criterion measure. Its drawback is that only principal vessels can be derived.

\section{6) segmentation using scale-space fuzzy connectedness}

Saha et al. [20] proposed scale-space fuzzy connectedness technique for vascular segmentation. The advantages of the method include: variable scales are defined locally to consider various sizes; the work can be extended to use other intensity-based features such as texture. However, user intervention is needed to select parameters.

7) segmentation using multiscale $3 D$ searching and region growing

Luo et al. [21] presented an automatic MRA cerebral vasculature segmentation algorithm with robust multiscale 3D searching and region growing. It consists of three major stages including 3D nonlinear anisotropic diffusion and noise removing; multiscale $3 \mathrm{D}$ vessel candidate searching; and vascular region growing. It is novel in multiscale $3 \mathrm{D}$ local searching based on both intensity gradient and intensity maxima. The algorithm also incorporates the knowledge of the morphology and topology of cerebral vasculature. Fig. 2 shows the three views of a vascular tree segmented with the proposed algorithm. The tree presents 
more than 100 cerebral vessels.

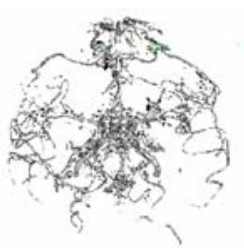

(a)

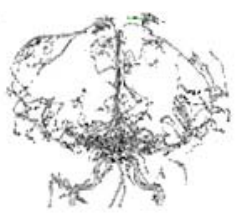

(b)

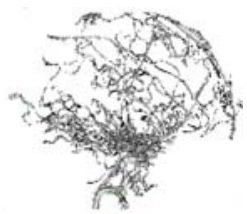

(c)
Fig. 2 Vascular tree segmented with the algorithm described in [xxx], on three views: (a) axial view, (b) coronal view, and (c) sagittal view.

\section{SUMMARY ON PROGRESS AND CHALLENGES AHEAD}

In the above review, the techniques discussed include: multiscale 3D searching and region growing; scale-space fuzzy connectedness; differential histogram and seeded region growing ; intensity ridge and Morse medialness; intensity correction, diffusion filtering and region growing; geodesic mathematical morphology and curvature evaluation; 3D multiscale line filter; watershed and neural networks; level sets; geodesic active contours; deformable model; locally adaptive region growing; and expectationmaximization. In the vascular segmentation literature, main issues mostly dealt with and emphasized on include: putting considerable consideration on the properties of cerebral vessels, especially their intensity and curvilinear structure, and segmenting vessels with various radii simultaneously.

Challenging issues of vessel segmentation that need to be paid much attention to include:

- Methodologies of validating the accuracy of vessel segmentation.

- Ways of hand-free: currently most of the approaches require users to manually input some parameters/thresholds.

- Ways of automatically deriving parameters locally as well as globally.

- Ways of connecting the vessel parts that were broken because of partial voluming, or filtering at segmenting.

- Ways of improving segmentation by introducing efficient image pre-processing and filtering such as locally adaptive techniques.

\section{OUR PROPOSALS AND CONCLUSION}

The above sections have presented a concise technical review on cerebral vasculature extraction from MRA, and detailed the main challenges to the researchers working in the area. Both fundamental segmentation techniques and the latest approaches are discussed. From the discussion, we reveal that the solution of efficient and practical head blood vessel segmentation cannot be resulted in from a conventional and straightforward approach, but in more advanced and hybrid approaches. In summary, the segmentation techniques that have shown potential and are worth paying attention to include: considering vessel's curvilinear structure; dealing with fine vessels as well as large ones; high automation in parameter selection; using local statistic information as well as global statistic information; detecting vessel linkage at weak places; and if the breakage can not recovered from intensity information, consider the incorporation of the morphologic and topologic knowledge of the cerebral vasculature.

Complete and automatic segmentation of the cerebral vasculature is still a long way to go. As more efforts are putting in the area, practical head vasculature segmentation systems will be available for clinic applications soon.

\section{REFERENCES}

[1] Potchen, E. J., et al., Magnetic Resonance Angiography: Concepts and Applications, Mosby-Year Book, Inc., 1993.

[2] Gonzalez, R. C., Woods, R. E., Digital Image Processing, Addison Wesley Publishing Company, Inc., 1993.

[3] Jadwiga Rogowska, Overview and fundamentals of medical image segmentation, Handbook of Medical Imaging Processing and Analysis Management, Editor-in-chief: Isaac Bankman, Academic Press, pp. 69-85, October 2000.

[4] Jasjit S. Suri, et al., Non-Skeleton and Skeleton-Based Segmentation Techniques from Angiography Data Sets, Angiography and Plaque Imaging: Advanced Segmentation Techniques, Ed. Swamy Laxminarayan, CRC Press, pp. 1-68, April 1, 2003.

[5] Jain, A. K., Fundamentals of Digital image Processing, Englewood Cliffs, NJ: Prentice Hall, 1989.

[6] Kirbas, C., and Quek, F. K. H., 2003. A Review of Vessel Extraction Techniques and Algorithms, Proc. Third IEEE Symposium on BioInformatics and BioEngineering BIBE 2003, pp. 238-245, 2003.

[7] Russ, J. C., The Image Processing Handbook, Boca Raton: CRC Press, 1999.

[8] Dempster, A.P., Laird, N.M., and Rubin, D.B., Maximum likelihood from incomplete data via the EM algorithm, J. Roy. Stat. Soc., 39, pp.1-38, 1977.

[9] Wilson, D.L., and Noble, J.A., Anadaptive segmentation algorithm for time-of-flight MRA data, IEEE Trans. Med. Imag., 18(10), pp.938945 , Oct. 1999.

[10] Yi, Jaeyoun, Ra, Jong Beom, A locally adaptive region growing algorithm for vascular segmentation, International Journal of Imaging Systems and Technology, 13(4), pp. 208-214, 2003.

[11] Kobashi, S., et al., Volume-quantization-based neural network approach to 3D MR angiography image segmentation, Image and Vision Computing, 19(4), pp. 185-193, , March 2001.

[12] Lorigo, L.M., et al., Co-dimension 2 geodesic active contours for MRA segmentation, Information Processing in Medical Imaging. 16th International Conference, IPMI'99, 1999, pp. 126-139.

[13] Farag, A. A., et al., 3D volume segmentation of MRA data sets using level sets, Academic Radiology, 11(4), pp. 419-435, April 2004.

[14] Hassan, H., and Farag, A. A., Cerebrovascular segmentation for MRA data using level sets, International Congress Series, 1256, pp. 246-252, June 2003.

[15] Yoshinobu Sato, et al., Three-dimensional multi-scale line filter for segmentation and visualization of curvilinear structures in medical images, Medical Image Analysis, 2(2), June 1998, pp.143-168.

[16] Frédéric Zana and Jean-Claude Klein, Segmentation of Vessel-Like Patterns Using Mathematical Morphology and Curvature Evaluation, IEEE Trans. on Image Processing, 10(7), July 2001.

[17] Flasque, N., et al., Acquisition, segmentation and tracking of the cerebral vascular tree on 3D magnetic resonance angiography images, Medical Image Analysis, 5(3), pp. 173-183, September 2001.

[18] Bullitt E., et al., Symbolic Description of Intracerebral Vessels Segmented from MRA and Evaluation by Comparison with X-Ray Angiograms, Medical Image Analysis 5:157-169, 2001.

[19] Tuduki, Y., et al., Automated seeded region growing algorithm for extraction of cerebral blood vessels from magnetic resonance angiographic data, Annual - Proceedings of Int. Conf. of the IEEE Engineering in Medicine and Biology, 3, pp. 1756-1759, 2000.

[20] Punam K. Saha, et al., Scale-Based Fuzzy Connected Image Segmentation: Theory, Algorithms, and Validation, Computer Vision and Image Understanding, 77(2), pp. 145-174, 2000.

[21] Luo, S, et al., Automatic MRA cerebral vasculature segmentation with robust multiscale 3D searching and region growing, The First Int. Conf. on Complex Medical Engineering, 2005, May 15-18, 2005. 